\title{
Eventos Extremos de Chuva no Estado do Rio Grande do Sul, Brasil, entre 2004 e 2013. Parte 2: Características Sinóticas dos Eventos Persistentes
}

\author{
Mateus da Silva Teixeira ${ }^{1}$ (D) , Raidel Báez Prieto $^{2}$ \\ ${ }^{1}$ Faculdade de Meteorologia, Universidade Federal de Pelotas, Pelotas, RS, Brasil. \\ ${ }^{2}$ Universidade Estadual de Santa Catarina, Florianópolis, SC, Brasil.
}

Recebido em: 7 de Março de 2019 - Aceito em: 31 de Julho de 2019

\begin{abstract}
Resumo
Nesta segunda parte do estudo sobre eventos persistentes de chuva são avaliadas as condições atmosféricas associadas aos eventos. Compostos de campos diagnósticos são apresentados para os eventos separados em função de sua duração. Eles mostram que sistemas atmosféricos com lento deslocamento propiciam a permanência de áreas de convergência do fluxo de umidade, transporte de calor e movimento vertical ascendente sobre o estado do Rio Grande do Sul por vários dias. A convergência do fluxo de umidade integrada verticalmente mostrou-se como uma boa ferramenta para inferência da importância dos eventos. Os campos diagnósticos apresentados não mostram uma indicação antecipada da duração de um evento de chuva, bem como não demostram com clareza a maior frequência de elevados acumulados de chuva na metade leste do estado do Rio Grande do Sul.
\end{abstract}

Palavras-chave: compostos, convergência fluxo de umidade, ciclogênese, frontogênese.

\section{Extreme rainfall events in the state of Rio Grande do Sul, Brazil, between 2004 and 2013. Part 2: synoptic characteristics of the persistent events}

\begin{abstract}
In this second part of the study about persistent rainfall events, the atmospheric conditions associated with these events are evaluated. Composites of diagnostic fields are presented for events classified by their duration. These composites showed that atmospheric systems with slow movement allow the permanence of areas of moisture flux convergence, heat transport and upward movement over the state of Rio Grande do Sul for several days. The moisture flux convergence vertically integrated proved to be a good tool to inference of the importance of events. The diagnostic fields did not show previously the duration of a rainfall event, as well as did not clearly demonstrate the major frequency of high rainfall accumulates in half east of the state of Rio Grande do Sul.
\end{abstract}

Keywords: composites, moisture flux convergence, ciclogenesis, frontogenesis.

\section{Introdução}

$\mathrm{Na}$ primeira parte desta investigação (Teixeira e Prieto, 2020), os eventos extremos persistentes e diários de chuva foram definidos e identificados no período de 2004 a 2013. Um total de 37 eventos persistentes foram identificados e 65 eventos diários. Foi observado que os eventos de chuva persistentes afetam com maior frequência a metade leste do estado do Rio Grande do Sul (RS), independente da duração.
Nesta segunda parte, propõe-se estudar o ambiente atmosférico dos eventos extremos persistentes de chuva ocorridos no estado do RS entre 2004 e 2013, procurando identificar os padrões associados a esses eventos. Além disso, a dinâmica atmosférica também será estudada, especialmente a dos dias anteriores e nos primeiros dias de duração dos eventos, bem como a associação dos padrões com a espacialização da chuva apresentada na primeira parte.

Autor de correspondência: Mateus da Silva Teixeira, mateus.teixeira@ufpel.edu.br. 
O conhecimento de padrões atmosféricos relacionados a eventos atmosféricos importantes é de suma importância à previsão de tempo, uma vez que pode colaborar para uma melhor indicação do potencial para esses eventos. Vários autores estudaram o ambiente sinótico relacionado a importantes eventos diários de chuva (p. ex., Severo, 1994; Harnack et al., 1999; Teixeira e Satyamurty, 2007; Lima et al., 2010; Moura et al., 2013) com o objetivo de obter padrões atmosféricos associados a esses eventos.

Tang et al. (2006) classificaram eventos persistentes de chuva ocorridos na China em função do escoamento em $500 \mathrm{hPa}$, dividindo-os em três grupos principais: (1) regime de oeste, (2) regime de leste e (3) convergência dos regimes de leste e oeste. $\mathrm{O}$ primeiro grupo conteve a maior parte dos eventos identificados. Zhao et al. (2017) distribuíram os eventos de chuva persistentes encontrados na China em categorias diferentes: (1) pré-estação de cheias no sul da China, (2) pós-estação de cheias no sul da China e (3) período da frente de Mei-yu, sendo o segundo aquele que possuiu mais eventos.

Prieto e Teixeira (2016) estudaram um evento de chuva persistente ocorrido no estado do RS em maio de 2004, em que algumas estações do leste do estado registraram chuvas superiores a $200 \mathrm{~mm}$, em 6 dias. Os autores verificaram que um lento processo de ciclogênese foi responsável pelas chuvas persistentes observadas. Zhang e Meng (2018) estudaram um evento persistente na China, que ocorreu entre 29 e 31 de março de 2014. Chuvas superiores a $200 \mathrm{~mm}$ foram observadas e estiveram associadas a um cavado de onda curta na média troposfera, um vórtice ciclônico em baixos níveis e a um jato de baixos níveis, que mantiveram uma linha de convergência persistente ao longo da costa sudeste da China.

\section{Materiais e Métodos}

Os eventos extremos de chuva ocorridos no estado do RS foram investigados por meio dos dados pluviométricos diários provenientes do Banco de Dados Meteorológicos para Ensino e Pesquisa (BDMEP) do Instituto Nacional de Meteorologia (INMET). Um total de 18 estações meteorológicas foram usadas. A distribuição espacial destas estações meteorológicas é apresentada na Fig. 1, de Teixeira e Prieto (2020).

As análises do modelo global GFS forneceram os dados das variáveis meteorológicas necessários à obtenção dos padrões associados aos eventos de chuva severa na região sul do Brasil (componentes zonal e meridional do vento, umidade específica, altura geopotencial etc). Estas análises têm resoluções espacial de $0,5^{\circ} \times 0,5^{\circ}$ de latitude e longitude, e temporal de 6 horas, disponíveis no National Centers for Environmental Information. Na vertical, estes dados estão distribuídos por 26 níveis, estendendo-se de 1000 a $10 \mathrm{hPa}$.
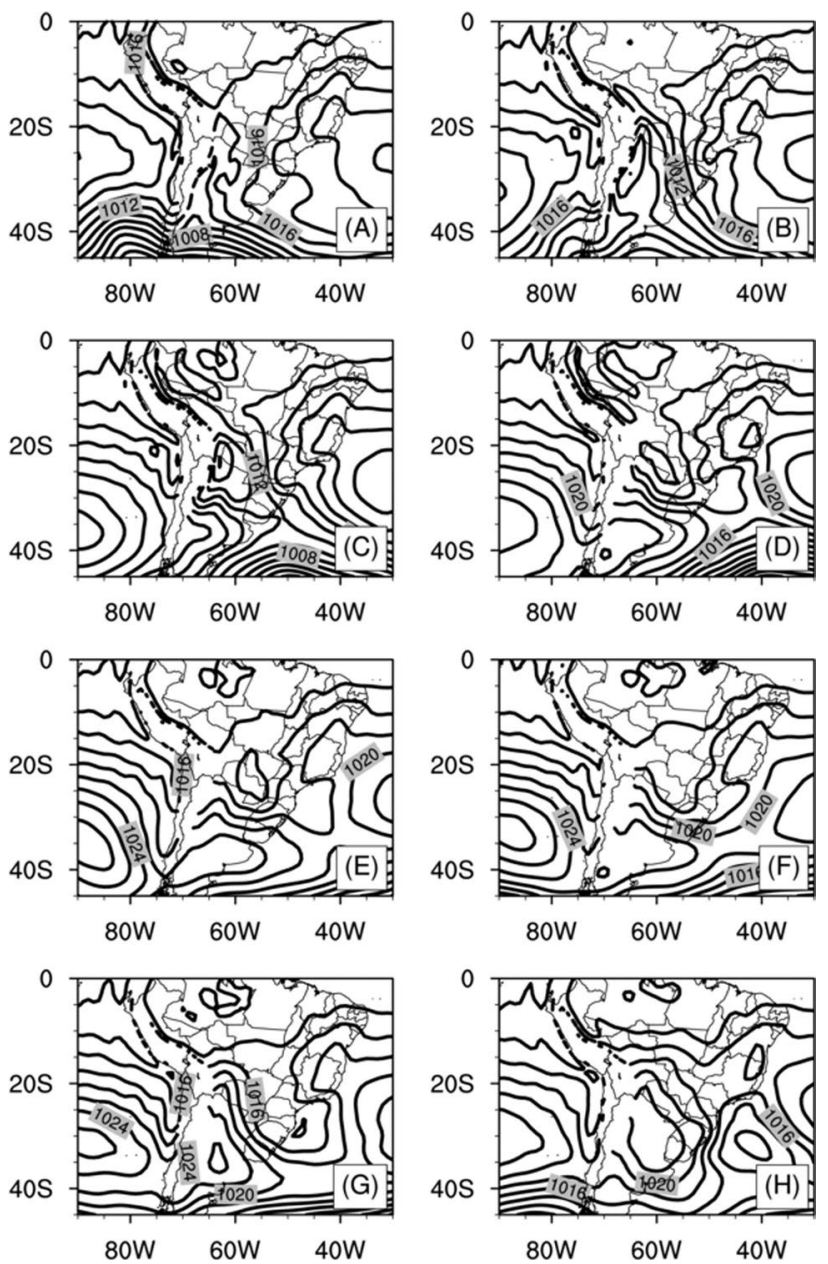

Figura 1 - Compostos de PNMM para os eventos com 5 dias de chuva persistente, sendo (A) para três dias antes do início da chuva (D-3), (B) para D-2, (C) para D-1, (D) para o dia do início da chuva (D0), (E) para um dia após o início da chuva (D1), $(\mathrm{F})$ para D2, $(\mathrm{G})$ para D3 e $(\mathrm{H})$ para D4. Intervalo das isóbaras de $2 \mathrm{hPa}$.

Os padrões foram obtidos por meio de campos médios, ou compostos, de pressão ao nível médio do mar (PNMM) para os eventos separados em função de sua duração. O estudo da dinâmica atmosférica associada aos eventos também será realizado por meio de compostos de outras variáveis e parâmetros meteorológicos, como advecção térmica em 850 e $500 \mathrm{hPa}$; convergência do fluxo de umidade em 850 , em $700 \mathrm{hPa}$ e integrada entre 1000 e $700 \mathrm{hPa}$; divergência do vento em 850 e $250 \mathrm{hPa}$; espessura 1000-500 hPa; linhas de corrente em 850 e $250 \mathrm{hPa}$; velocidade vertical (ômega) em 850 e $500 \mathrm{hPa}$; advecção de vorticidade relativa em 850 e $500 \mathrm{hPa}$ e advecção vertical de umidade.

Conforme mostrado na primeira parte deste estudo (Teixeira e Prieto, 2020, Tabela 1) os eventos persistentes selecionados têm durações que variam de 5 a 14 dias, sendo que apenas um evento teve duração de 14 dias. Para permitir uma comparação entre os diferentes eventos, os 
campos compostos foram obtidos desde três dias antes do início do período de 24 horas de chuva (D-3) até o quinto dia de duração do evento (D4), sendo que o início é considerado como D0. Fica evidente que para os casos com duração de 5 dias, o dia D4 refere-se ao último dia do evento, mas não para os demais. Então, a comparação entre os diferentes grupos de eventos fica prejudicada para o dia $\mathrm{D} 4$, visto que as condições atmosféricas permanecem favorecendo a ocorrência de chuva nos eventos com mais de 5 dias. Os campos apresentados referem-se ao horário das 12 UTC.

\section{Resultados e Discussões}

A Fig. 1 mostra os compostos de pressão ao nível médio do mar (PNMM) para os 10 eventos que tiveram 5 dias de duração. A maioria dos eventos -5 eventos ocorreram durante o inverno (JAS). Primavera e outono tiveram dois eventos, cada, e o verão apenas um. Pode-se observar nos campos apresentados que 3 dias antes (Fig. 1-A) do início dos eventos um cavado sobre o sul da Argentina e Chile, desconfigurado pela sua passagem pela Cordilheira dos Andes. Nos dias seguintes, ele se desloca para leste concomitante à formação de uma área de baixa pressão sobre o norte da Argentina (Figs. 1-A-C). Um dia antes do início dos eventos (D-1; Fig. 1C), pode-se observar uma região de colo sobre a região do Uruguai e RS. Regiões de colo estão associadas a calmaria, mas mais especialmente, à deformação no campo de vento horizontal. A deformação no campo de escoamento horizontal tem um papel importante no processo de frontogênese (Bluestein, 1992) e como a maioria dos casos de chuva persistente com 5 dias de duração foram identificados no inverno ( $50 \%$ dos casos), essa característica no campo de PNMM pode ter colaborado para a formação de sistemas frontais sobre a região do Uruguai e RS. A orientação zonal do movimento ascendente na região (Fig. 3D) evidencia essa possibilidade. Zhang e Meng (2018) também observaram uma área de colo na região na região de ocorrência de um evento de chuva persistente ocorrido na China, em março de 2014. No início dos eventos (D0; Fig. 1-D) essa área de baixa pressão está localizada sobre o Paraguai, mas influencia o estado do RS (e boa parte da região sul do Brasil) com escoamento predominante de norte. Posteriormente, essa região de baixa pressão, especialmente até o D2 (Figs. 1-E e F), tem pequeno deslocamento, mantendo a sua influência sobre a região. Nos dias seguintes, (Figs. 1-G e H) a área de baixa pressão ganha forma de um ciclone e se desloca em direção ao Oceano Atlântico (OA). No último dia (Fig. 1-H), a região da Argentina, Paraguai e RS estão sob a atuação de um centro de alta pressão.

Os compostos de altura geopotencial em $500 \mathrm{hPa}$ para esses eventos mostram um incremento no gradiente horizontal de altura geopotencial na parte sul do continen- te sul-americano, desde dois dias antes do início dos eventos (D-2; Fig. 2-B). Um dia antes, o gradiente era mais fraco e bastante zonal (D-3; Fig. 2-A). Isso evidencia, também, um aumento no gradiente térmico médio da camada sobre essas regiões, indicativo de um processo baroclínico, possivelmente associado ao processo de frontogênese em superfície. Nos dias seguintes, o cavado em níveis médios se desloca para leste. Concordando com o apresentado nos compostos de PNMM, o cavado tem um deslocamento relativamente lento nos primeiros dias dos eventos persistentes de chuva. A partir do dia D2 (Fig. 2F) esse cavado parece se deslocar mais rapidamente, em relação aos dias anteriores, uma vez que no dia D4 ele já se encontra sobre a costa do RS. Esse padrão é muito similar aquele encontrado para a maioria dos eventos (grupos sob o regime de oeste em $500 \mathrm{hPa}$ ) de chuva persistentes estudados por Tang et al. (2006), que também observaram um cavado em níveis médios. Lynch e Schumacher (2014) e Zhang e Meng (2018) também verificaram a presença de cavados em níveis médios associados a eventos de chuva persistente nos Estados Unidos e na
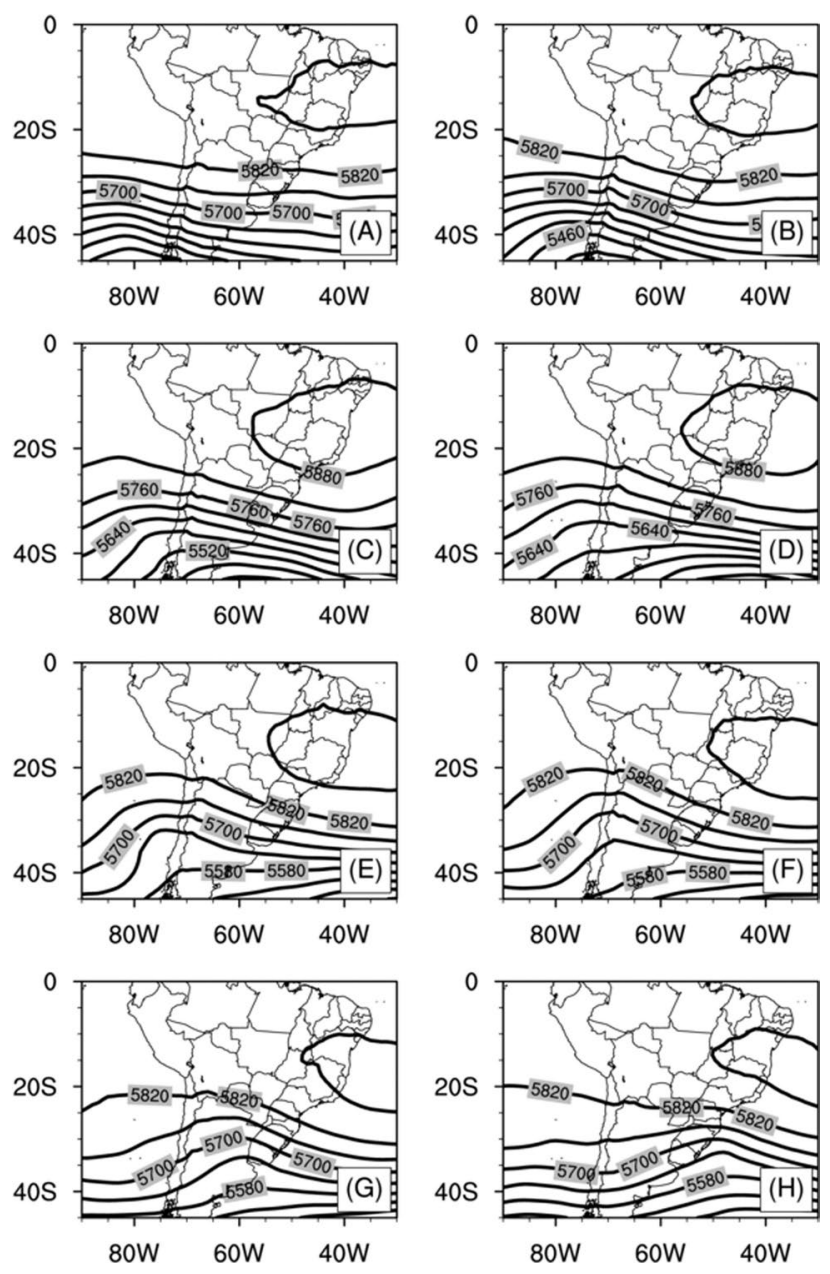

Figura 2 - Idem a Fig. 1, mas para os compostos de altura geopotencial em $500 \mathrm{hPa}$. Intervalo das isoípsas de $60 \mathrm{mgp}$. 
China, respectivamente. É interessante ressaltar uma aparente "quebra" no cavado, que pode ser observada entre D2 e D-1. Nessa "quebra", a parte sul desse cavado, localizada nas latitudes médias se desloca para leste mais rapidamente que a sua parte norte. Esse comportamento é refletido em superfície, onde se observa um ciclone nas latitudes médias (aproximadamente $50^{\circ} \mathrm{S}$ ) se deslocando rapidamente para leste, enquanto os processos de frontogênese e ciclogênese ocorrem sobre o continente.

A Fig. 3 apresenta os campos compostos da velocidade vertical omega em $500 \mathrm{hPa}$, em que são destacados apenas os valores negativos, correspondentes a movimento vertical ascendente. Estes compostos mostram, claramente, o efeito da evolução observada nos campos de PNMM e altura geopotencial em $500 \mathrm{hPa}$, pois no dia do início dos eventos (D0; Fig. 3-D) tem-se movimentos ascendentes em praticamente todo o estado do RS, efeito da aproximação dos cavados em superfície e em níveis médios. Nos dias anteriores (D-3 a D-1; Figs. 3-A até C) pode-se acompanhar a aproximação da região de movimento ascendente ao estado. Nos dias seguintes ao início
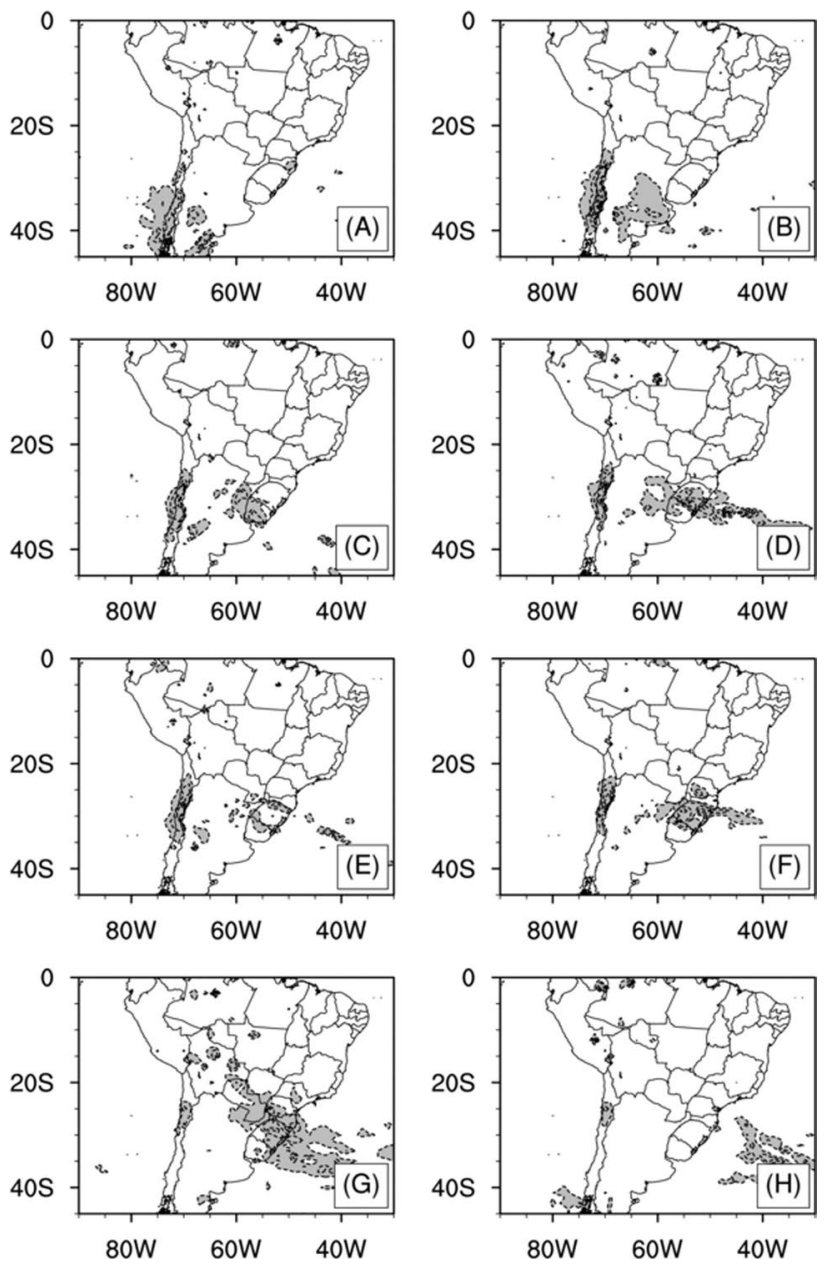

Figura 3 - Idem a Fig. 1, mas para os compostos de velocidade vertical ômega em $500 \mathrm{hPa}$. Intervalo dos contornos de $0,2 \mathrm{~Pa} \mathrm{~s}^{-1}$. dos eventos, pode-se perceber o efeito do lento deslocamento do sistema atmosférico, que permitiu a permanência de movimento ascendente por praticamente 4 dias. No quinto dia, a área de ascensão encontra-se sobre o OA. Apesar do campo composto para o quinto dia não evidenciar movimento ascendente sobre a região, é importante enfatizar que a chuva registrada no quinto dia de chuva refere-se à chuva acumulada entre 12 UTC do quarto dia e 12 UTC do quinto dia. Como os campos se referem às 12 UTC do dia em questão, o quinto dia acaba mostrando o final do evento, a partir do qual não se tem mais chuvas na região.

As Figs. 4 a 6 mostram o resultado da organização do escoamento atmosférico conforme os campos apresentados nas figuras anteriores. Pode-se perceber que o
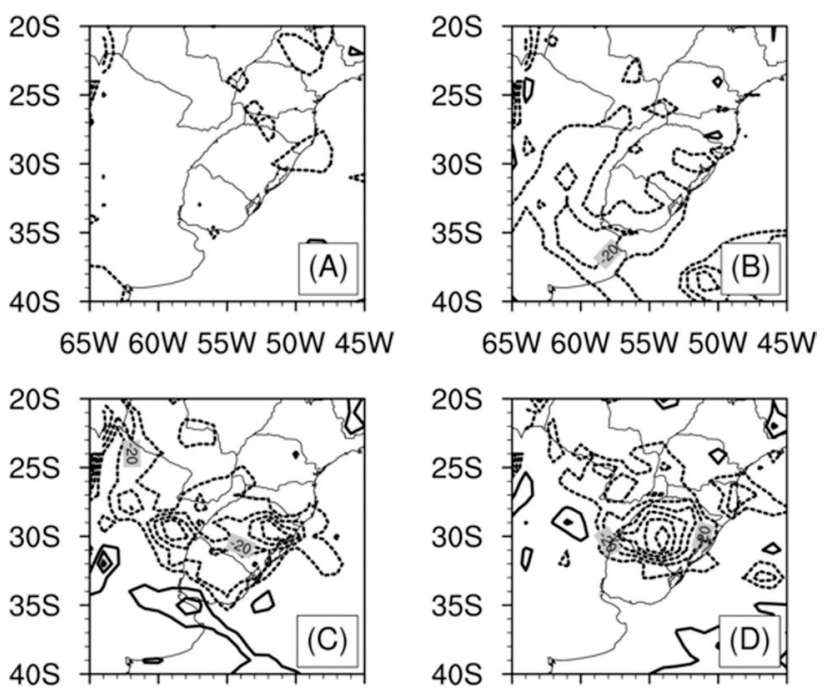

65W 60W 55W 50W 45W
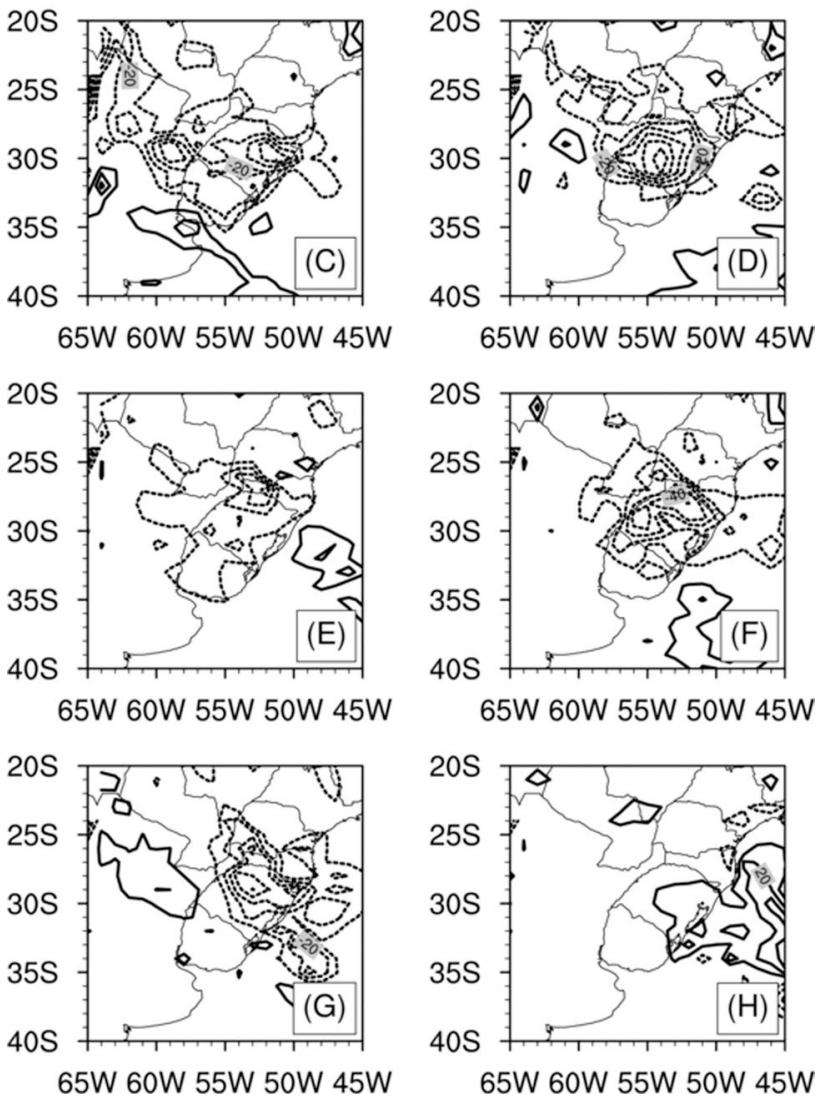

Figura 4 - Idem a Fig. 1, mas para a divergência horizontal do fluxo de umidade integrada na vertical, entre os níveis de 1000 e $700 \mathrm{hPa}$. Intervalo dos contornos de $10^{-4} \mathrm{~kg} \mathrm{~m}^{-2} \mathrm{~s}^{-1}$. 

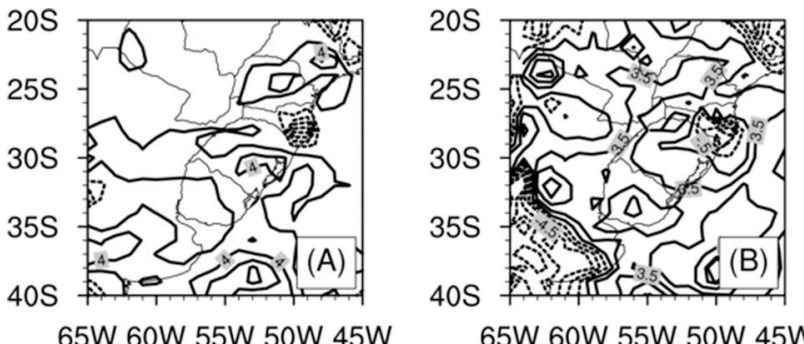

65W 60W 55W 50W 45W

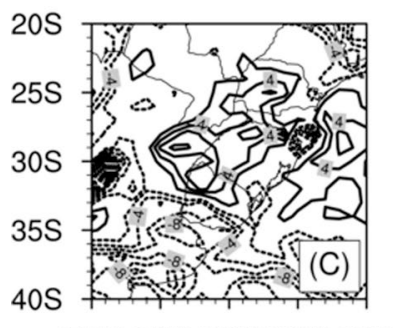

65W 60W 55W 50W 45W

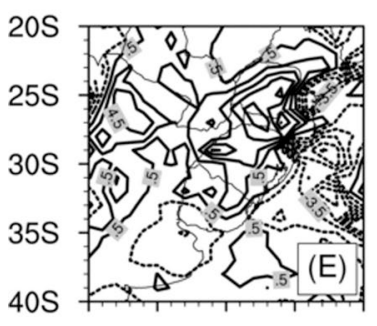

65W 60W 55W 50W 45W

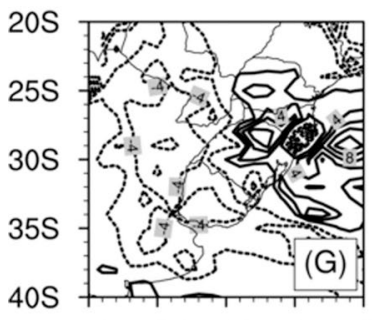

65W 60W 55W 50W 45W
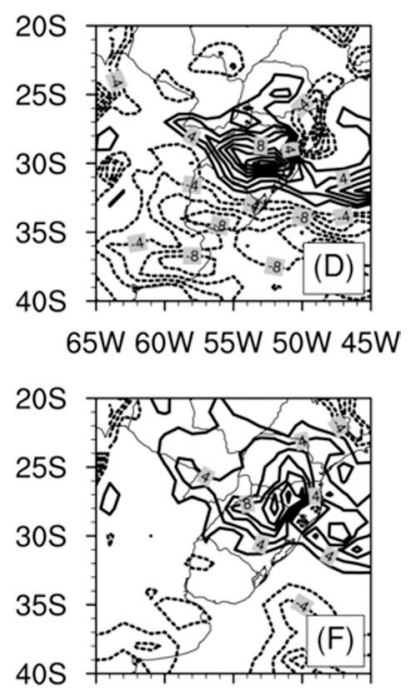

65W 60W 55W 50W 45W

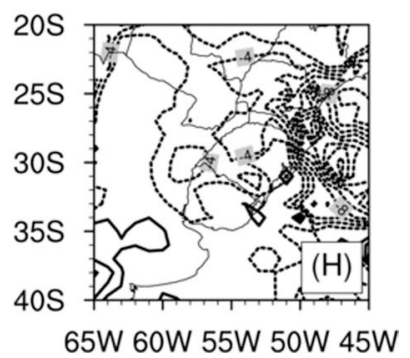

Figura 5 - Idem a Fig. 1, mas para a advecção de temperatura do ar em $850 \mathrm{hPa}$. Intervalo dos contornos de $2 \times 10^{-5} \mathrm{~K} \mathrm{~s}^{-1}$.

escoamento confluente sobre a região (Fig. 6) propiciou uma convergência do fluxo de umidade na baixa troposfera, na camada 1000-700 hPa, desde o dia D-2 (Fig. 4B), mantendo-se até o dia D3 (Fig. 4-G). A presença de movimento vertical ascendente e convergência de umidade na camada mais baixa da troposfera propiciou a ocorrência da chuva observada nos eventos identificados. Morales e Yamasaki (2016) mostraram que os campos prognósticos de convergência do fluxo de umidade verticalmente integrada são úteis na previsão de tempestades, uma vez que concordam com a localização das nuvens observadas posteriormente, especialmente sobre o continente, nas latitudes médias.

O escoamento em baixos níveis (Fig. 6) também favoreceu o transporte de ar quente dos trópicos para o RS (Fig. 5). Após o término da chuva na região pode-se per-
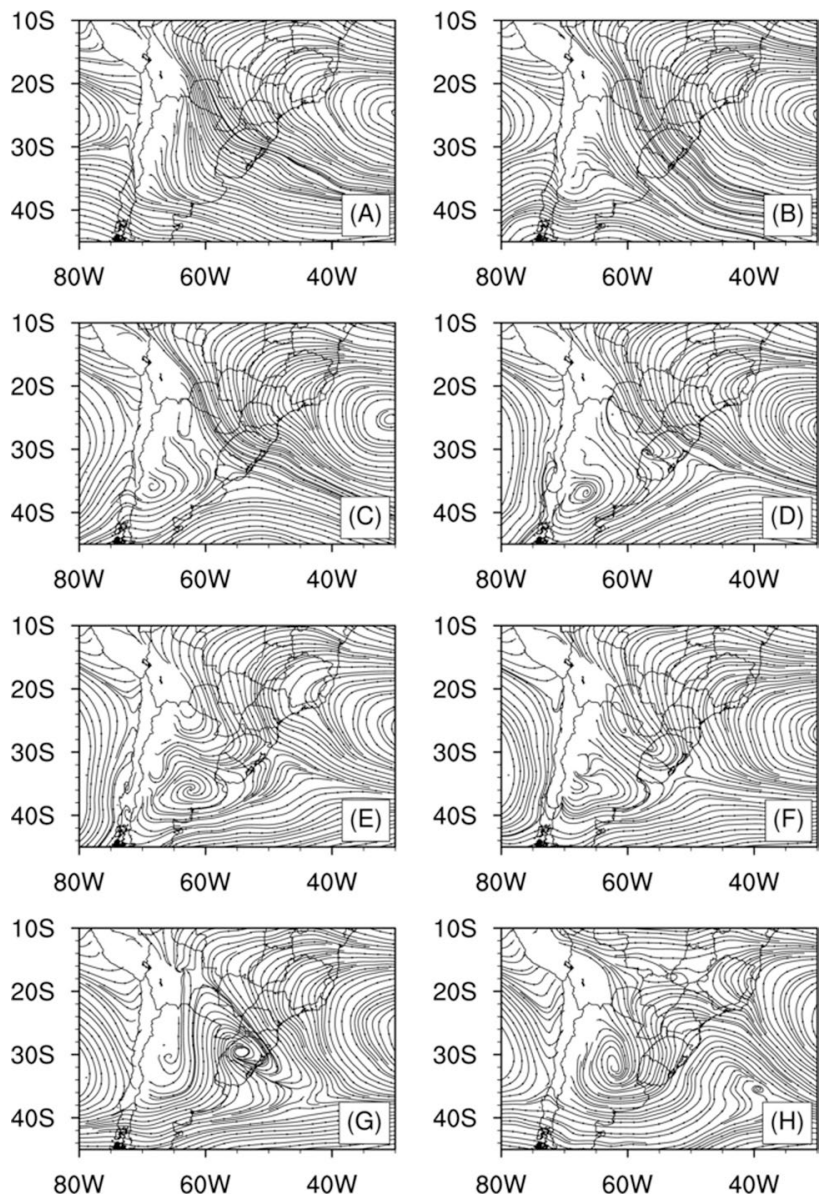

Figura 6 - Idem a Fig. 1, mas para as linhas de corrente em $850 \mathrm{hPa}$.

ceber um transporte de ar relativamente mais frio, quando o escoamento de sul predomina sobre o estado do RS (Fig. 5-H). É interessante notar que não apenas o estado do RS estava sob influência do transporte de ar quente, mas toda a região sul do Brasil. Entretanto, a convergência do fluxo de umidade esteve mais concentrada sobre o RS, o que colaborou para que a chuva fosse mais intensa sobre a região de estudo. Lynch e Schumacher (2014) e Zhang e Meng (2018) ressaltaram a presença de um jato de baixos níveis como componente crucial para o transporte de umidade para as regiões de chuva persistente estudadas por eles.

Assim, pela evolução dos campos de PNMM e altura geopotencial em $500 \mathrm{hPa}$ - e dos demais campos diagnósticos - pode-se observar que nos eventos com 5 dias de chuva um processo de frontogênese e posterior ciclogênese sobre o Uruguai e RS foram as principais causas para que o transporte de umidade e o movimento ascendente persistisse sobre a região por vários dias.

Outro ponto interessante a ser notado refere-se ao escoamento em baixos níveis. É esperado em resultados obtidos por compostos, ou seja, médias simples de campos atmosféricos, que certas características sejam "suaviza- 
das" ao longo do domínio em estudo, mostrando uma aparência mais branda e mais generalizada sobre a área avaliada - muito em função das diferentes posições dos sistemas entre os diferentes casos envolvidos no cômputo do campo composto. Entretanto, os compostos para os casos com até 5 dias de duração mostram com clareza uma ciclogênese sobre o estado do RS e seu posterior deslocamento para o oceano, com a entrada de um anticiclone. Essa característica dos compostos, em especial dos compostos de PNMM (Fig. 1) e de linhas de corrente (Fig. 6), evidenciam que, apesar de possíveis diferenças de posicionamento entre os 10 eventos com 5 dias de duração, as diferenças não foram grandes o suficiente para "mascarar" os aspectos importantes do escoamento atmosférico e suas consequências relacionados aos casos estudados.

A Fig. 7 mostra a evolução temporal da divergência horizontal do vento média na área do estado do RS, em vários níveis verticais. As linhas tracejadas em preto mostram os dias antes do início da chuva, sendo que a linha tracejada mais espessa marca o dia D-3. A linha cheia marca o dia D0, o início da chuva, e as demais linhas em cinza mostram os dias seguintes, sendo aquela mais espessa o último dia, D4. Pode-se ver que em praticamente todos os dias, a metade mais baixa da troposfera esteve sob influência de convergência do vento (valores negativos). Apenas no último dia, quando a chuva cessa sobre o estado, há divergência em toda essa camada, inibindo movimentos ascendentes, como pode ser evidenciado na Fig. 3-G.

Outro fator interessante na avaliação da divergência horizontal média é a presença de máximos de convergência no nível de $900 \mathrm{hPa}$ de D-3 até D0 e no nível de $850 \mathrm{hPa}$ durante os dias seguintes. É comum tomar o nível

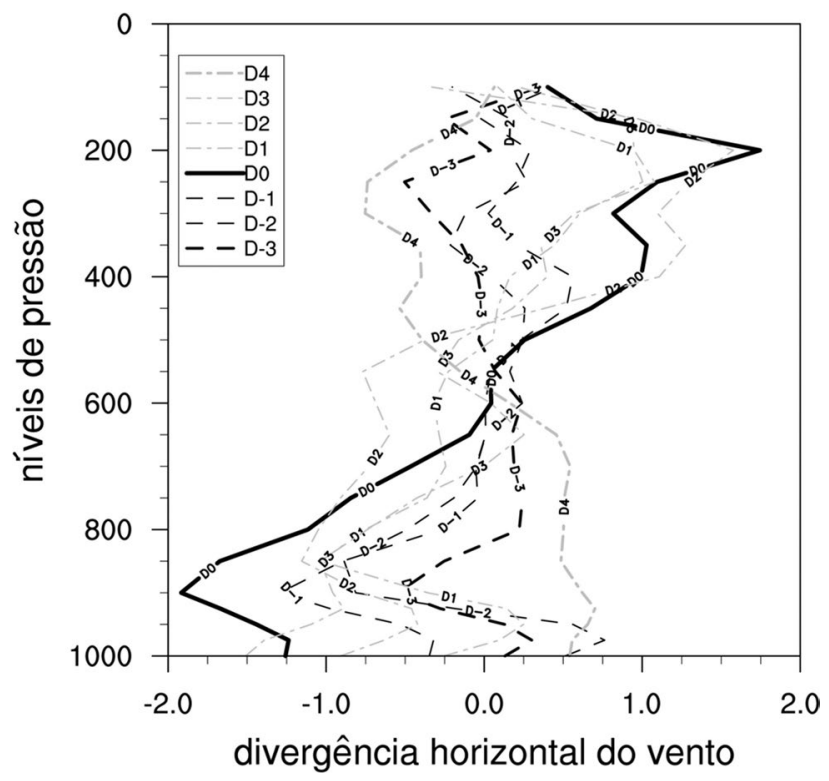

Figura 7 - Evolução temporal da distribuição vertical de divergência horizontal do vento. Os valores, em $10^{-5} \mathrm{~s}^{-1}$, são médios na área do estado do RS. de $850 \mathrm{hPa}$ como referência para avaliações de transporte e convergência de umidade. Mostra-se, dessa forma, a importância da avaliação integrada na vertical dos diagnósticos a respeito da umidade atmosférica, seja do seu transporte (muitas vezes relacionado aos "rios atmosféricos"), seja da divergência e convergência desse transporte, e não apenas em um único nível (Zhu e Newell, 1998; Banacos e Schultz, 2005; Morales e Yamasaki, 2016). Por exemplo, Lavers e Villarini (2013) mostraram que os rios atmosféricos são determinantes para a ocorrência de chuva extrema na Europa Ocidental. E na América do Sul, a região a leste da Cordilheira dos Andes é o local de ocorrência de um dos mais importantes rios atmosféricos deste continente: o Jato de Baixos Níveis da América do Sul (JBNAS; Marengo et al., 2004). O início do evento (D0) é marcado por um máximo de convergência em baixos níveis e um máximo de divergência em altos níveis, em $200 \mathrm{hPa}$. Conforme o passar dos dias, esses máximos diminuem, invertendo o sinal no último dia.

A Fig. 8 mostra os campos compostos de PNMM dos 14 casos com 6 dias de duração. Por questões de comparação, é mostrado a mesma quantidade de dias mostrada para
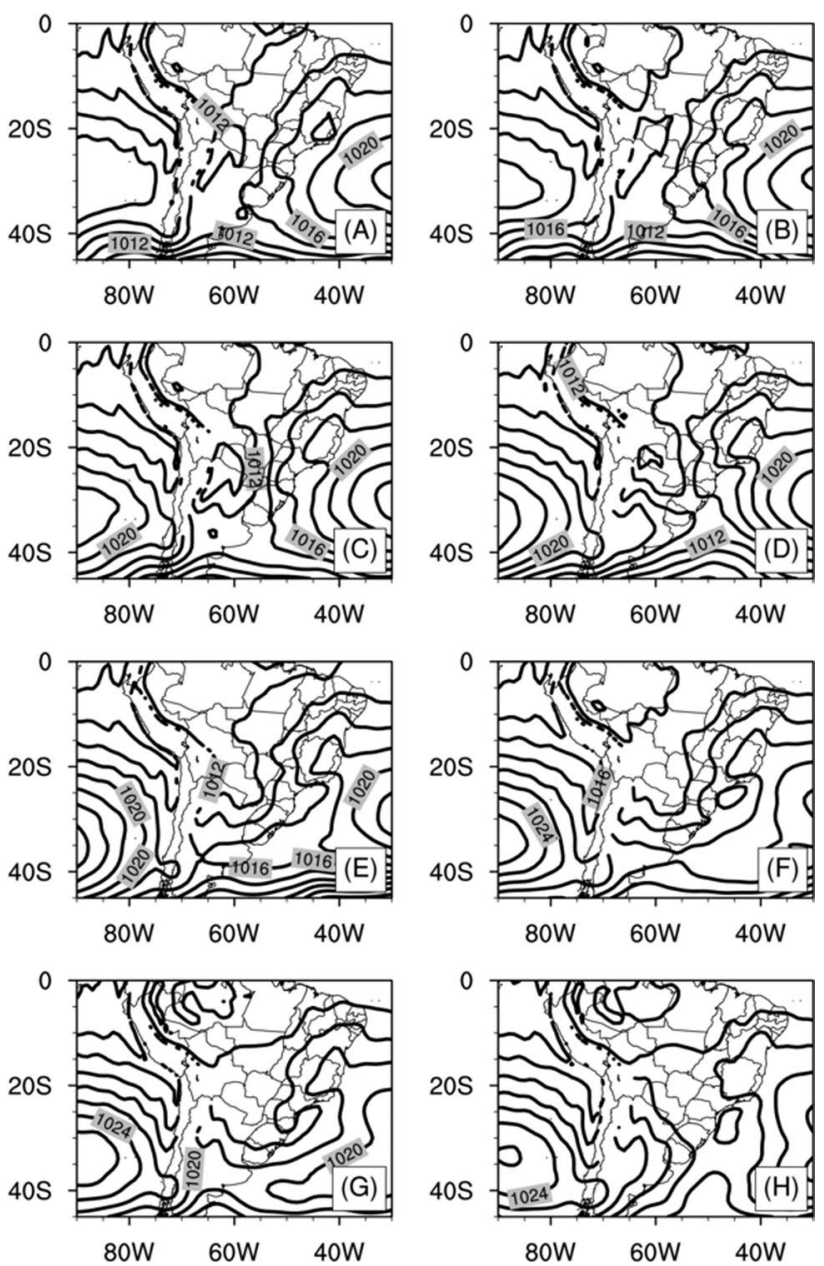

Figura 8 - Idem Fig. 1, mas para os eventos com 6 dias de duração. 
os casos com 5 dias de duração. Em outras palavras, os campos de D-3 até D4 são apresentados. Pode-se ver, em comparação com os campos da Fig. 1, que tiveram uma evolução similar, exceto pela aparente formação de um ciclone sobre o oceano, na costa do estado de São Paulo (SP). Entretanto, não é possível perceber uma circulação ciclônica nos compostos desses eventos (Fig. 9), como evidenciado nos eventos com um dia a menos de duração (Fig. 6). Mesmo assim, pode-se perceber um escoamento com forte componente de norte por praticamente todo o período. Em níveis médios (não mostrado) não há uma configuração importante, sendo observado um cavado de baixa intensidade sobre o oeste do continente sul-americano em D0 e que avança para leste nos dias seguintes, perdendo intensidade. Também em níveis médios, o movimento ascendente é observado em D0 (não mostrado), com abrangência menor na região de estudo. Também se observa nesses casos uma região de colo sobre o estado do RS (Fig. 8D), mas não surge como um precursor do evento de chuva, como visto anteriormente, pois essa configuração é percebida em D0, no início do evento de chuva.

A Fig. 10 mostra a divergência horizontal integrada na camada 1000-700 hPa, cujos campos evidenciam a pre-
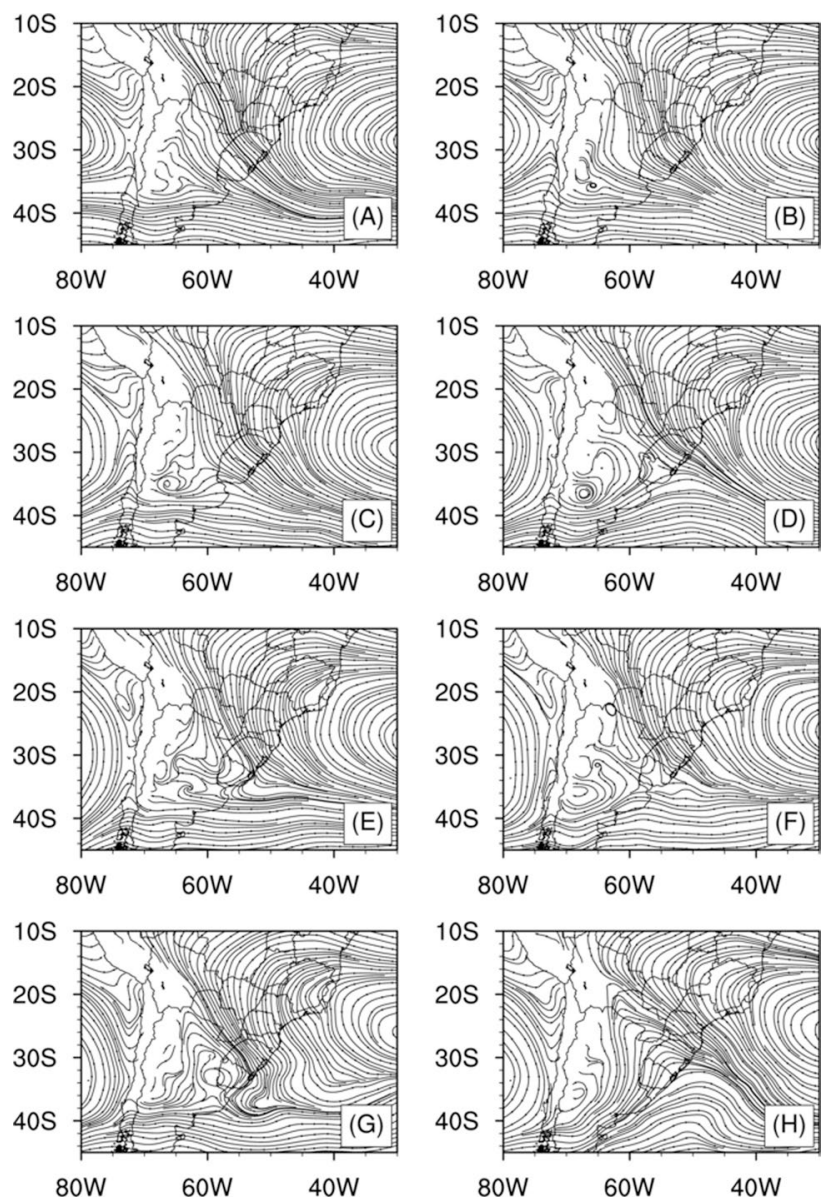

Figura 9 - Idem Fig. 6, mas para os eventos com 6 dias de duração. sença de convergência sobre a região desde D-1. Nos eventos com 5 dias de duração essa convergência era percebida já em D-2. Além disso, parece possuir intensidade menor, em comparação com o outro conjunto de eventos. A advecção de ar quente para a região também é abrangente (não mostrado) como nos compostos para os casos com 5 dias de duração, mas é mais fraca (praticamente a metade, em intensidade). A ocorrência de uma ciclogênese na costa da região sudeste do Brasil, portanto mais ao norte da observada nos compostos para os eventos com 5 dias de duração, pode ter sido a causa para um escoamento menos intenso e, consequentemente, num transporte mais fraco de ar quente e úmido para o RS.

A Fig. 11 mostra a evolução temporal da divergência do vento para os casos com 6 dias de duração. Ela corrobora o que foi discutido acima, a respeito da intensidade

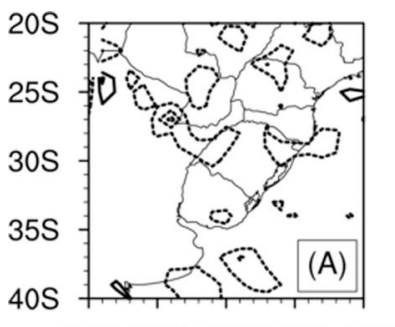
65W 60W 55W 50W 45W
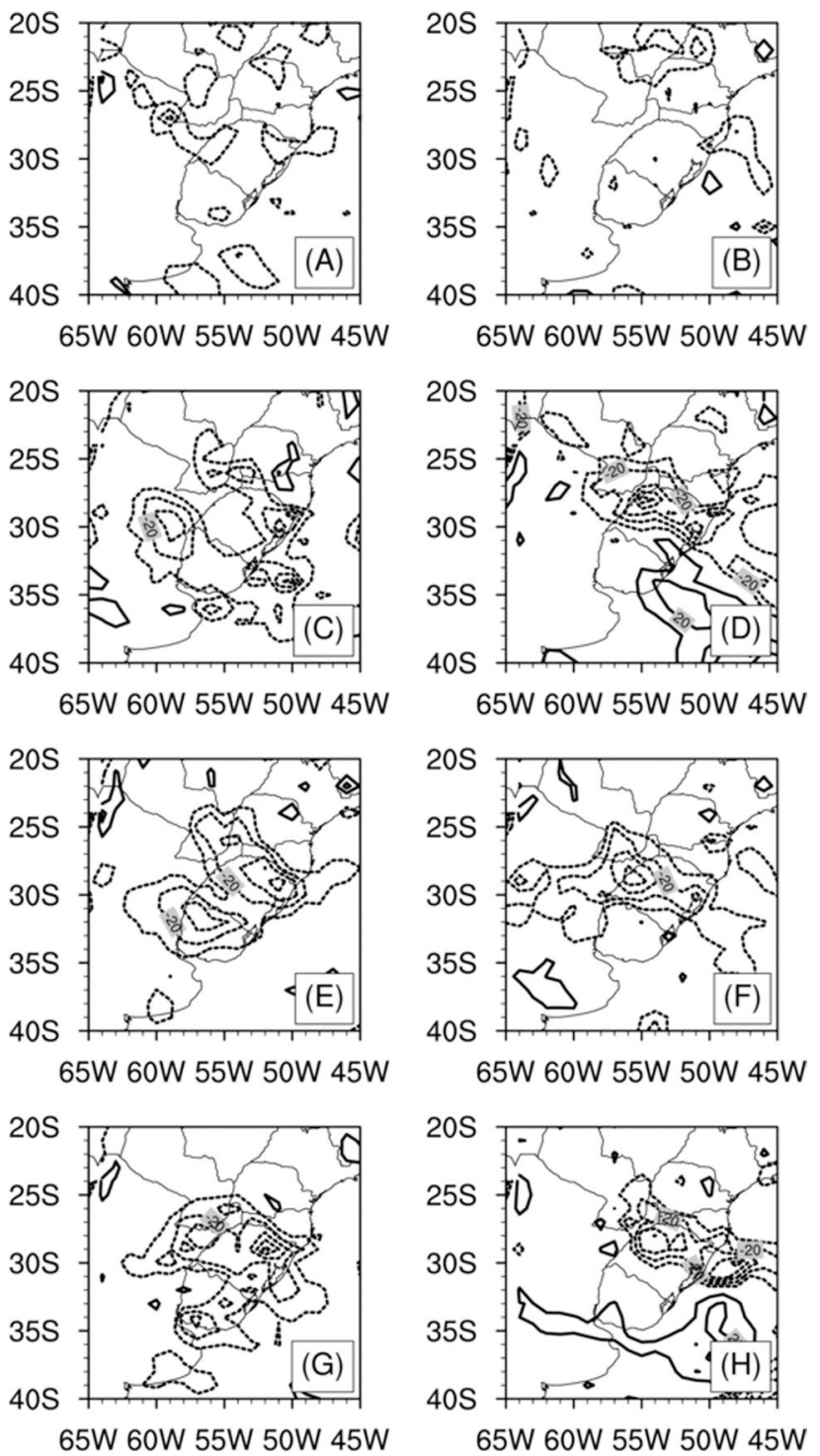

Figura 10 - Idem Fig. 4, mas para os eventos com 6 dias de duração. 


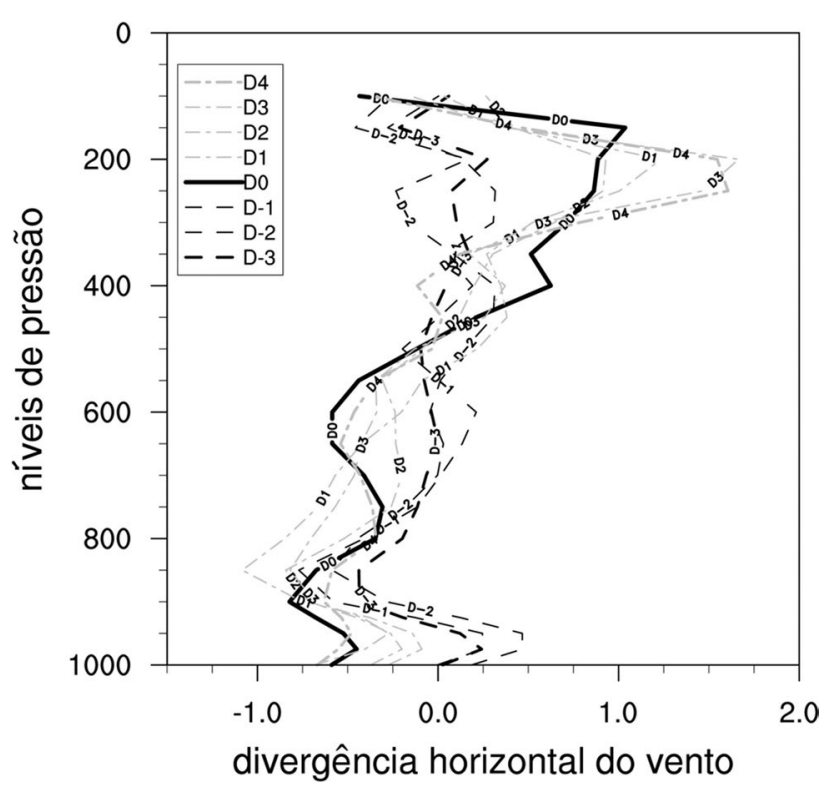

Figura 11 - Idem a Fig. 7, mas para os eventos com 6 dias de duração.

dos campos diagnósticos. Em comparação com os eventos com 5 dias de duração, a intensidade da convergência na baixa troposfera é menor.

Os compostos para as demais durações não são apresentados de maneira detalhada, pois possuem uma quantidade menor de eventos. Essa menor quantidade pode prejudicar a comparação, uma vez que as magnitudes dos campos tendem a ser menos suavizadas. Entretanto, uma comparação "mais subjetiva" revela evoluções similares ao longo do tempo analisado - de D-3 a D4.

Nos campos avaliados não foi possível identificar alguma característica que ajude a identificar se um evento de chuva terá uma certa quantidade maior de dias de chuva. Ou seja, não foi possível determinar padrões que diferencie substancialmente eventos de menor duração dos eventos com maior duração. Igualmente, não é possível estabelecer uma relação direta entre a espacialização da chuva apresentada na primeira parte deste estudo com os campos diagnósticos. A convergência do fluxo de umidade integrada verticalmente mostra núcleos intensos nas partes norte e nordeste do RS, mas é difícil a relação direta entre esses núcleos e uma maior frequência de chuvas mais intensas na metade leste do estado. Morales e Yamasaki (2016) associaram essa convergência integrada verticalmente com aglomerados de nuvens que causaram chuva intensa, mas não diretamente com a chuva intensa.

Assim, apesar dos campos diagnósticos não conseguirem mostrar algum sinal de distinção entre casos de diferentes durações, acredita-se que os campos aqui apresentados possam ser comparados com as saídas de modelos numéricos de previsão de tempo para identificar se os eventos previstos poderão se tornar duradouros.

\section{Conclusões}

A partir dos resultados obtidos, pode-se concluir que:

- Os processos de frontogênese e ciclogênese proporcionaram a permanência de convergência do fluxo de umidade, advecção de calor e movimento ascendente sobre o estado do RS por vários dias.

- Como sugerido por outros estudos, o uso da convergência do fluxo de umidade integrada na vertical é recomendado, pois os casos persistentes apresentaram máximos dessa convergência abaixo do nível de $850 \mathrm{hPa}$, referência para diagnósticos na baixa troposfera.

- A espacialização da chuva realizada na primeira parte deste estudo não teve uma comprovação direta a partir dos campos diagnósticos, que mostrasse porque a metade leste do RS tem maiores quantidades de chuva associadas aos eventos persistentes.

- Não há como distinguir a duração de um evento de chuva apenas pelas características da atmosfera próxima à região da AS. É provável que uma avaliação mais profunda da atmosfera sobre os oceanos ajude sobremaneira nessa tarefa.

Há vários tópicos que ainda permanecem não respondidos a respeito dessa classe de eventos de chuva. Portanto, futuras investigações continuarão a ser realizadas com o objetivo de entender melhor os motivos pelos quais tais chuvas ocorrem por períodos prolongados no estado do RS.

\section{Referências}

BANACOS, P.C.; SCHULTZ, D.M. The use of moisture flux convergence in forecasting convective initiation: historical and operational perspectives. Weather and Forecasting, v. 20, n. 3, p. 351-366, 2005.

BLUESTEIN, H. Synoptic-Dynamic Meteorology in Midlatitudes. Oxford University Press, New York, p. 448, 1992.

HARNACK, R.P.; APEL, K.; CERMAK, J.R. Heavy precipitation events in New Jersey: Attendant upper air conditions. Weather and Forecasting, v.14, n. 6, p. 933-954, 1999.

LAVERS, D.A.; VILLARINI, G. The nexus between atmospheric rivers and extreme precipitation across Europe. Geophysical Research Letters, v. 40, n. 12, p. 3259-3264, 2013.

LIMA, K.C.; SATYAMURTY, P.; FERNÁNDEZ, J.P.R. Largescale atmospheric conditions associated with heavy rainfall episodes in southeast brazil. Theoretical and Applied Climatology, v. 101, n. 1-2, p. 121-135, 2010.

LYNCH, S.L.; SCHUMACHER, R.S. Ensemble-based analysis of the May 2010 extreme rainfall in Tennessee and Kentucky. Monthly Weather Review, v. 142, n. 1, p. 222-239, 2014.

MARENGO, J.A.; SOARES, W.R.; SAULO, C.; NICOLINI, M. Climatology of the low-level jet east of the Andes as derived from the NCEP-NCAR reanalyses: characteristics 
and temporal variability. Journal of Climate, v. 17, n. 12, p. 2261-2280, 2004.

MORALES, W.; YAMASAKI, Y. Análise da convergência do fluxo de umidade verticalmente integrada sobre o Brasil previsto pelo modelo WRF. Ciência e Natura, v. 38, n. 1, p. 371-381, 2016.

MOURA, C.R.W.; ESCOBAR, G.C.J.; ANDRADE, K.M. Padrões de circulação em superfície e altitude associados a eventos de chuva intensa na região metropolitana do Rio de Janeiro. Revista Brasileira de Meteorologia, v. 28, n. 3, p. 267-280, 2013.

PRIETO, R.B.; TEIXEIRA, M.S. Análisis sinóptico de um evento extremo y persistente de lluvia sobre el estado de Río Grande do Sul, Brasil, en mayo de 2004. Ciência e Natura, v. 38, n. 2, p. 1110-1115, 2016.

SEVERO, D.L. Estudo de Casos de Chuvas Intensas no Estado de Santa Catarina. 1994. 118 p. Dissertação de Mestrado em Meteorologia, Instituto Nacional de Pesquisas Espaciais, São José dos Campos.

TEIXEIRA, M.S.; PRIETO, R.B. Eventos extremos de chuva no estado do Rio Grande do Sul, Brasil, entre 2004 e 2013. Parte 1: definição dos eventos e estatísticas. Revista Brasileira de Meteorologia, v. 35, n. 1, p. 53-61, 2020.

TEIXEIRA, M.S.; SATYAMURTY, P. Dynamical and synoptic characteristics of heavy rainfall episodes in southern Brazil. Monthly Weather Review, v. 135, n. 2, p. 598-617, 2007.
ZHANG, J.; LI, L.; ZHOU, T.; XIN, X. Evaluation of spring persistent rainfall over East Asia in CMIP3/CMIP5 AGCM simulations. Advances in Atmospheric Science, v. 30, n. 6, p. 1587-1600, 2013.

ZHANG, M.; MENG, Z. Impact of synoptic-scale factors on rainfall forecast in different stages of a persistent heavy rainfall in South China. Journal of Geophysical Research: Atmosphere, v. 123, n. 7, p. 3574-3593, 2018.

ZHAO, Y.; WANG, D.; LIANG, Z.; XU, J. On the dynamics of the large-scale circulation during the persistent severe rainfall events in Southern China. Journal of the Meteorological Society of Japan, v. 95, n. 2, p. 111-125, 2017.

ZHU, Y.; NEWELL, R.E. A proposed algorithm for moisture fluxes from atmospheric rivers. Monthly Weather Review, v. 126, n. 3 , p. $725-735,1998$.

\section{Endereços de Internet}

National Centers for Environmental Information, https://www. ncdc.noaa.gov/data-access/model-data.

License information: This is an open-access article distributed under the terms of the Creative Commons Attribution License (type CC-BY), which permits unrestricted use, distribution and reproduction in any medium, provided the original article is properly cited. 